\title{
Higher-form symmetry breaking at Ising transitions
}

\author{
Jiarui Zhao $\odot,{ }^{1}$ Zheng Yan, ${ }^{1,2}$ Meng Cheng, ${ }^{3, *}$ and Zi Yang Meng $\oplus^{1, \dagger}$ \\ ${ }^{1}$ Department of Physics and HKU-UCAS Joint Institute of Theoretical and Computational Physics, The University of Hong Kong, \\ Pokfulam Road, Hong Kong SAR, China \\ ${ }^{2}$ State Key Laboratory of Surface Physics and Department of Physics, Fudan University, Shanghai 200438, China \\ ${ }^{3}$ Department of Physics, Yale University, New Haven, Connecticut 06520-8120, USA
}

(Received 2 December 2020; revised 18 May 2021; accepted 19 May 2021; published 6 July 2021)

\begin{abstract}
In recent years, new phases of matter that are beyond the Landau paradigm of symmetry breaking have been accumulating, and to catch up with this fast development, new notions of global symmetry are introduced. Among them, the higher-form symmetry, whose symmetry charges are spatially extended, can be used to describe topologically ordered phases as the spontaneous breaking of the symmetry, and consequently unify the unconventional and conventional phases under the same conceptual framework. However, such conceptual tools have not been put into quantitative tests except for certain solvable models, therefore limiting their usage in the more generic quantum many-body systems. In this work, we study $\mathbb{Z}_{2}$ higher-form symmetry in a quantum Ising model, which is dual to the global (zero-form) Ising symmetry. We compute the expectation value of the Ising disorder operator, which is a nonlocal order parameter for the higher-form symmetry, analytically in free scalar theories and through unbiased quantum Monte Carlo simulations for the interacting fixed point in $(2+1) d$. From the scaling form of this extended object, we confirm that the higher-form symmetry is indeed spontaneously broken inside the paramagnetic, or quantum disordered phase (in the Landau sense), but remains symmetric in the ferromagnetic or ordered phase. At the Ising critical point, we find that the disorder operator also obeys a "perimeter" law scaling with possibly multiplicative power-law corrections. We discuss examples where both the global zero-form symmetry and the dual higher-form symmetry are preserved, in systems with a codimension-1 manifold of gapless points in momentum space. These results provide nontrivial working examples of higher-form symmetry operators, including the direct computation of one-form order parameter in an interacting conformal field theory, and open the avenue for their generic implementation in quantum many-body systems.
\end{abstract}

DOI: 10.1103/PhysRevResearch.3.033024

\section{INTRODUCTION}

Global symmetries are instrumental in organizing our understanding of phases of matter. The celebrated Landau paradigm classifies phases according to broken symmetries, which also determines the universality classes of transitions between phases. Symmetry principles become even more powerful from the point of view of long-wavelength, lowenergy physics, as the renormalization group fixed points (i.e., IR) often embody more symmetries than the microscopic lattice model (i.e., UV), which is the phenomenon of emergent symmetry [1-5]. A common example is the emergence of continuous space-time symmetries in the field-theoretical description of a continuous phase transition [6]. It is even plausible that a critical point is determined up to finite choices

\footnotetext{
*m.cheng@yale.edu

†zymeng@hku.hk
}

Published by the American Physical Society under the terms of the Creative Commons Attribution 4.0 International license. Further distribution of this work must maintain attribution to the author(s) and the published article's title, journal citation, and DOI. by its full emergent symmetry, which is the basic philosophy (or educated guess) behind the conformal bootstrap program [7].

Modern developments in quantum many-body physics have significantly broadened the scope of quantum phases beyond the Landau classification [8]. For these exotic phases, more general notions of global symmetry are called for to completely characterize the phases and the associated phase transitions. Intuitively, these "beyond Landau" phases do not have local order parameters. Instead, nonlocal observables are often needed to characterize them. For a well-known example, confined and deconfined phases of a gauge theory are distinguished by the behavior of the expectation value of Wilson loop operators $[9,10]$. To incorporate such extended observables into the symmetry framework, higher-form symmetries [11-13], and more generally algebraic symmetries [14,15], have been introduced. These are symmetries whose charged objects are spatially extended, e.g., strings and membranes. In other words, their symmetry transformations only act nontrivially on extended objects. Most notably, spontaneous breaking of such higher symmetries can lead to highly entangled phases, such as topological order [13]. Therefore, even though topologically ordered phases are often said to be beyond the Landau paradigm, they can actually be understood within a 
similar conceptual framework once higher symmetries are included. In addition, just as the usual global symmetries, higher-form symmetries can have quantum anomalies [13], which lead to strong nonperturbative constraints on lowenergy dynamics [16].

In this work, we make use of the prototypical continuous quantum phase transition, the Ising transition, to elucidate the functionality of the higher-form symmetry. The motivation to reexamine the well-understood Ising transition is the following: in addition to the defining zero-form $\mathbb{Z}_{2}$ symmetry, the topological requirement that $\mathbb{Z}_{2}$ domain walls must be closed (in the absence of spatial boundary) can be equivalently formulated as having an unbreakable $\mathbb{Z}_{2}(D-1)$-form symmetry, where $D$ is the spatial dimension. The gapped phase on either side of the transition spontaneously breaks one and only one of the two symmetries. Therefore, to correctly determine the full emergent internal symmetry in the Ising conformal field theory (CFT), the $\mathbb{Z}_{2}$ higher-form symmetry should be taken into account. For $D=2$, the 1-form symmetry manifests more clearly in the dual formulation [17], namely, as the confinement-deconfinement transition of a $\mathbb{Z}_{2}$ gauge theory, which will shed light on higher-form symmetry-breaking transitions in a concrete setting.

A basic question about a global symmetry is whether it is broken spontaneously or not in the ground state. For clarity, let us focus on the $D=2$ case. It is well known that the Ising symmetric, or "quantum disordered," phase spontaneously breaks the higher-form symmetry, and the opposite in the Ising symmetry-breaking phase. The fate at the critical point remains unclear to date. To diagnose higher-form symmetry breaking, we compute the ground-state expectation value of the "order parameter" for the higher-form symmetrycommonly known as the disorder operator in the literature [18-22], which creates a domain wall in the Ising system. Spontaneous breaking of the $\mathbb{Z}_{2} 1$-form symmetry is signified by the perimeter law for the disorder operator. In the dual formulation, the corresponding object is the Wilson loop operator. Through large-scale quantum Monte Carlo (QMC) simulations, we find numerically that at the transition, the disorder operator defined on a rectangular region scales as $l^{s} e^{-a_{1} l}$, where $l$ is the perimeter of the region, and $s>0$ is a universal constant. We thus conclude that the 1-form symmetry is spontaneously broken at the $(2+1) d$ Ising transition, and it remains so in the disordered phase of the model. This is in stark contrast with the $D=1$ case, where the disorder operator has a power-law decay.

To corroborate the numerical results, we consider generally a disorder operator corresponding to a zero-form $\mathbb{Z}_{2}$ symmetry in a free scalar theory in $D$ dimensions, which is a stable fixed point for $D \geqslant 3$. We show that for the kind of $\mathbb{Z}_{2}$ symmetry in this case, the disorder operator can be related to the second Rényi entropy. Therefore, the disorder operator also obeys a "perimeter" (i.e., volume of the boundary) scaling, with possibly multiplicative power-law correction. Whether the higher-form symmetry is broken or not is determined by the subleading power-law corrections. We also discuss other free theories, such as a Fermi liquid, where the decay of the disorder operator is in between the "perimeter" and the "area" laws, and therefore there is no higher-form symmetry breaking.
The rest of the paper is organized as follows. In Sec. II we review higher-form symmetry and its spontaneous breaking, and its relevance in conventional phases. We also consider higher-form symmetry breaking in free and interacting conformal field theories. In Sec. III we specialize to the setting of the quantum Ising model in $(2+1) d$ and define the disorder operator. Section IV presents the main numerical results from quantum Monte Carlo simulations, which reveal the key evidence of the 1 -form symmetry breaking at the $(2+1) d$ Ising transition. Section $\mathrm{V}$ outlines a few immediate directions about the higher-form symmetry breaking and their measurements in unbiased numerical treatments in other quantum many-body systems.

\section{GENERALIZED GLOBAL SYMMETRY}

Consider a quantum many-body system in $D$ spatial dimensions. Global symmetries are unitary transformations which commute with the Hamiltonian. Typically the symmetry transformation is defined over the entire system, and charges of the global symmetry are carried by particlelike objects.

An important generalization of global symmetry is the higher-form symmetry [13]. For an integer $p \geqslant 0, p$-form symmetry transformations act nontrivially on $p$-dimensional objects. In other words, "charges" of $p$-form symmetry are carried by extended objects. In this language, the usual global symmetry is zero-form as the particlelike object is of zero dimension. The $p$-form symmetry transformations themselves are unitary operators supported on each codimension- $p$ [i.e., spatial dimension $(D-p)]$ closed submanifold $M_{D-p}$. In particular, it means that there are infinitely many symmetry transformations in the thermodynamic limit. In this work we will only consider discrete, Abelian higher-form symmetry, so for each submanifold $M_{D-p}$ the associated unitary operators form a finite Abelian group $G$. Physically, higher-form symmetry means that the certain $p$-dimensional objects are charged under the group $G$, and the quantum numbers they carry constrain the processes of creation, annihilation and splitting, etc. In particular, these extended objects are "unbreakable"; i.e., they are always closed and cannot end on $(p-1)$-dimensional objects.

For a concrete example, let us consider $(2+1) d \mathbb{Z}_{2}$ gauge theory defined on a square lattice. Each edge of the lattice is associated with a $\mathbb{Z}_{2}$ gauge field (i.e., a qubit), subject to Gauss's law at each site $v$ :

$$
\prod_{e \ni v} \tau_{e}^{x}=1
$$

Here $e$ runs over edges ending on $v$.

The divergence-free condition implies that there are no electric charges in the gauge theory. In other words, all $\mathbb{Z}_{2}$ electric field lines must form loops. An electric loop can be created by applying the following operator along any closed path $\gamma$ on the lattice:

$$
W_{e}(\gamma)=\prod_{e \in \gamma} \tau_{e}^{z} .
$$




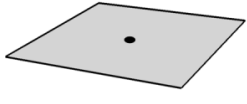

(a)

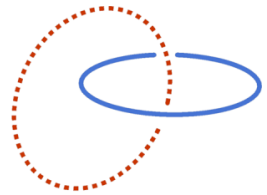

(b)
FIG. 1. (a) The zero-form symmetry charge is a pointlike object, measured by the symmetry transformation defined on the entire system (i.e., at a fixed time slice). (b) The 1-form symmetry charge is a loop (the solid line), e.g., $W_{e}$, measured by the symmetry transformation defined on a loop (dotted line), e.g., $W_{m}$, when the two loops are linked.

The corresponding $\mathbb{Z}_{2} 1$-form symmetry operator is defined as

$$
W_{m}\left(\gamma^{\star}\right)=\prod_{e \perp \gamma^{\star}} \tau_{e}^{x}
$$

for any closed path $\gamma^{\star}$ on the dual lattice. Here the subscript $m$ in $W_{m}$ indicates that this is actually the string operator for $\mathbb{Z}_{2}$ flux excitations. In field theory parlance, $W_{e}$ is the Wilson operator of the $\mathbb{Z}_{2}$ gauge theory, and $W_{m}$ is the corresponding Gukov-Witten operator [23].

We notice that the $W_{m}\left(\gamma^{\star}\right)$ operator is in fact the product of Gauss's law term $\prod_{v \in e} \tau_{e}^{x}$ for all $v$ in the region enclosed by $\gamma^{\star}$. In other words, the smallest possible $\gamma^{\star}$ is a loop around one vertex $v$, and the fact that $W_{m}\left(\gamma^{\star}\right)$ is conserved by the dynamics means that the gauge charge at site $v$ must be conserved $(\bmod 2)$ as well. Therefore, the $\mathbb{Z}_{2}$ gauge theory with electric 1-form symmetry is one with completely static charges, including the case with no charges at all. For applications in relativistic quantum field theories, it is usually further required that the 1-form symmetry transformation is "topological," i.e., not affected by local deformation of the loop $\gamma^{\star}$, which is equivalent to the absence of gauge charge as given in Eq. (1).

It is instructive to consider how the 1-form charge of an electric loop can be measured. This is most clearly done in space-time: to measure a $p$-dimensional charge, one "wraps" around the charge by a $(D-p)$-dimensional symmetry operator. Applying the symmetry transformation is equivalent to shrinking the symmetry operator, and in $(D+1)$ space-time because of the linking the two must collide, and the noncommutativity (e.g., between $W_{e}$ and $W_{m}$ ) measures the charge value. We illustrate the process for $p=0$ [Fig. 1(a)] and $p=1$ [Fig. 1(b)], in three-dimensional space-time.

Now consider the following Hamiltonian of Ising gauge theory:

$$
H=-J \sum_{e} \tau_{e}^{x}-K \sum_{p} \prod_{e \in \partial p} \tau_{e}^{z},
$$

where $J, K>0$. When $J \ll K$, the ground state is in the deconfined phase, which can be viewed as an equal-weight superposition of all closed $\mathbb{Z}_{2}$ electric loops. In this phase, the $\mathbb{Z}_{2} 1$-form symmetry is spontaneously broken. When $J \gg K$, the ground state is a product state with $\tau_{e}^{x}=1$ everywhere, and the 1-form symmetry is preserved. This is the confined phase. Similar to the usual boson condensation, the expec- tation value of the electric loop creation operator $W_{e}(\gamma)$ can be used to characterize the 1-form symmetry-breaking phase, which obeys perimeter law in the deconfined phase.

This example shows that higher-form symmetry naturally arises in gauge theories. In condensed matter applications, gauge theories are usually emergent [3,24], which means that dynamical gauge charges are inevitably present and the electric 1-form symmetry is explicitly broken. Even under such circumstances, at energy scales well below the electric charge gap, the theory still has an emergent 1-form symmetry [25].

Let us now discuss more generally the spontaneous breaking of higher-form symmetry [13,26,27]. We will assume that the symmetry group is discrete. For a $p$-form symmetry, a charged object is created by an extended operator $W(C)$ defined on a $p$-dimensional manifold $C$. When the symmetry is unbroken, we have

$$
\langle W(C)\rangle \sim e^{-t_{p+1} \operatorname{Area}(C)},
$$

where $\operatorname{Area}(C)$ is the volume of a minimal $(p+1)$ dimensional manifold whose boundary is $C . t_{p+1}$ can be understood as the "tension" of the $(p+1)$-dimensional manifold. This generalizes the exponential decay of the charged local operator for the zero-form case. On the other hand, when the symmetry is spontaneously broken,

$$
\langle W(C)\rangle \sim e^{-t_{p} \text { Perimeter }(C)},
$$

where Perimeter $(C)$ denotes the "volume" of $C$ itself. Importantly the expectation value only depends locally on $C$, which is the analog of the factorization of the correlation function of local order parameter $\left\langle O(x) O^{\dagger}(y)\right\rangle \approx\langle O(x)\rangle\left\langle O^{\dagger}(y)\right\rangle$ for zeroform symmetry. One can then redefine the operator $W(C)$ to remove the perimeter scaling and in that case $\langle W(C)\rangle$ would approach a constant in the limit of large $C$ [28]. At a critical point, however, subleading corrections become important, which will be examined below.

The $\mathbb{Z}_{2}$ gauge theory is famously dual to a quantum Ising model [29]. In fact, more generally, there is a duality transformation which relates a system with global $\mathbb{Z}_{2}$ zero-form symmetry (in the $\mathbb{Z}_{2}$ even sector) to one with global $\mathbb{Z}_{2}(D-$ 1)-form symmetry, a generalization of the Kramers-Wannier duality in $(1+1) d$.

Let us now review the duality in $(2+1) d$. The dual Ising spins are defined on plaquettes, whose centers form the dual lattice. For a given edge $e$ of the original lattice, we denote the two adjacent plaquettes by $p$ and $q$, as shown in Fig. 2 .

The duality map is defined as follows:

$$
\sigma_{p}^{z} \sigma_{q}^{z} \leftrightarrow \tau_{e}^{x}, \sigma_{p}^{x} \leftrightarrow \prod_{e \in \partial p} \tau_{e}^{z} .
$$

Note that the expression automatically ensures $\prod_{p} \sigma_{p}^{x}=1$ in a closed system, so the dual spin system has a $\mathbb{Z}_{2}$ zero-form symmetry generated by $S=\prod_{p} \sigma_{p}^{x}$, and the map can only be done in the $\mathbb{Z}_{2}$ even sector with $S=1 .{ }^{1}$ Conversely, the mapping also implies $\prod_{v \in e} \tau_{x}^{e}=1$, and in fact $W_{m}\left(\gamma^{\star}\right)=1$ for any $\gamma^{*}$, i.e., the $\mathbb{Z}_{2} 1$-form symmetry is strictly enforced.

\footnotetext{
${ }^{1}$ In a sense the $\mathbb{Z}_{2}$ symmetry is gauged. In fact one way to derive the duality is to first gauge the $\mathbb{Z}_{2}$ symmetry and then perform gauge transformations to eliminate the Ising matter.
} 


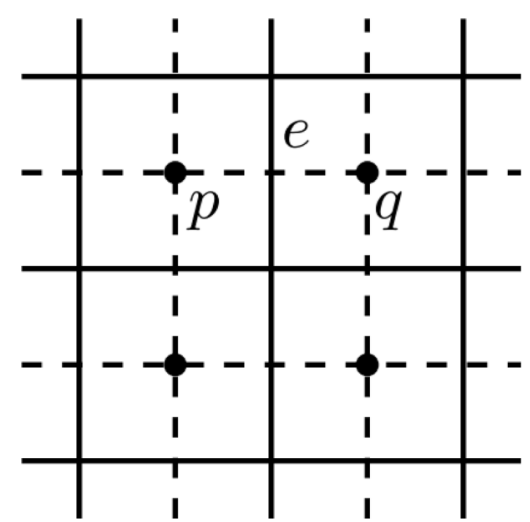

FIG. 2. The square lattice (solid line) and its dual lattice (dashed line). $\mathbb{Z}_{2}$ gauge fields live on the edges (e.g., $e$ in the figure) of the original lattice, and the dual Ising spins live on the dual lattice (e.g., square plaquette centers $p, q$ ).

In the dual model, the electric field line of the $\mathbb{Z}_{2}$ gauge theory becomes the domain walls separating regions with opposite Ising magnetizations. Therefore, a Wilson loop $W_{e}(\gamma)$ maps to

$$
X_{M}=\prod_{p \in M} \sigma_{p}^{x},
$$

where $\partial M=\gamma$, i.e., $M$ is the region enclosed by $\gamma$. Physically $X_{M}$ flips all the Ising spins in the region $M$, thus creating a domain wall along the boundary $\gamma$. It is called the disorder operator for the Ising system, which will be the focus of our study below.

Under the duality map, the Hamiltonian becomes

$$
H=-J \sum_{\langle p q\rangle} \sigma_{p}^{z} \sigma_{q}^{z}-K \sum_{p} \sigma_{p}^{x} .
$$

The phases of the gauge theory can be readily understood in the dual representation. For $K \gg J$, the $\mathbb{Z}_{2}$ gauge theory is in the deconfined phase, which means that the ground state contains arbitrarily large electric loops. For the dual Ising model, the ground state is disordered, with all $\sigma_{p}^{x}=1$. If we work in the $\sigma^{z}$ eigenbasis (which is natural to discuss symmetry breaking), the ground-state wave function is given by

$$
\left|\psi_{K=\infty}\right\rangle \propto \prod_{p} \frac{1+\sigma_{p}^{x}}{2}|\uparrow \uparrow \cdots \uparrow\rangle .
$$

Namely, we pick any basis state and apply the ground-state projector. Expanding out the projector, one can see that the wave function is an equal superposition of all domain wall configurations, i.e., a condensation of domain walls. Since the domain walls carry $\mathbb{Z}_{2} 1$-form charges, the condensation breaks the 1-form symmetry spontaneously, much like the Bose condensation spontaneously breaks the conservation of particle numbers.

In the other limit $K \ll J$, the gauge theory is confined. Correspondingly, the dual Ising model is in the ferromagnetically ordered phase: there are two degenerate ground states $|\uparrow \cdots \uparrow\rangle$ and $|\downarrow \cdots \downarrow\rangle$. There are no domain walls at all in the limit $K \rightarrow 0$. When a small but finite $K / J$ is turned on, quantum fluctuations create domain walls on top of the fully polarized ground states, but these domain walls are small and sparse.

\section{A. Noninvertible anomaly and gapless states}

A notable feature of the duality map is that on either side, only one of two symmetries, the $\mathbb{Z}_{2}$ zero-form and the $\mathbb{Z}_{2}$ 1 -form symmetries, is faithfully represented (in the sense that the symmetry transformation is implemented by a nontrivial operator, even though the duality is supposed to work only in the symmetric sector). The other symmetry transformation is mapped to the identity at the operator level. Physically, only one of them is an explicit global symmetry, while the other one appears as a global constraint [e.g., on the Ising side, domain walls of the zero-form global symmetry are codimension-1 closed manifolds, which is the manifestation that they are charged under a $(D-1)$-form symmetry].

A closely related fact is that the ordered phase for one symmetry is necessarily the disordered phase of the other, and any nondegenerate gapped phase must break one and only one of the two symmetries. This has been proven rigorously in one spatial dimension [30], and is believed to hold in general dimensions as well.

It is clear from these results that these two symmetries cannot be considered as completely independent. Recently, Ref. [31] proposed that the precise relation between the two dual symmetries is captured by the notion of a noninvertible quantum anomaly. Intuitively, the meaning of the noninvertible anomaly in the context of the $\mathbb{Z}_{2}$ Ising model can be understood as follows: the charge of the $\mathbb{Z}_{2}$ zero-form symmetry is an Ising spin flip, while the charge of the $\mathbb{Z}_{2} 1$-form symmetry is an Ising domain wall. These two objects have nontrivial mutual "braiding" in the sense that when an Ising charge is moved across a domain wall, it picks up a minus sign due to the Ising symmetry transformation applied to one side of the domain wall. In other words, the charge of the 1-form symmetry is actually a flux loop of the zero-form symmetry. Reference [31] suggested that two symmetries whose charged objects braid nontrivially with each other cannot be realized faithfully in a local Hilbert space. If locality is insisted, then the only option is to realize the $D$ spatial dimensional system as the boundary of a $\mathbb{Z}_{2}$ toric code model in $(D+1)$ spatial dimensions. In this case, the charged objects are in fact bulk topological excitations brought to the boundary. The nontrivial braiding statistics between the two kinds of charges reflects the topological order in the bulk. Such an anomaly is fundamentally different from the more familiar 't Hooft anomaly realized on the boundary of a symmetry-protected topological phase (which is an invertible state). We refer to Ref. [31] for more thorough discussions of the noninvertible anomaly.

Since any gapped state must break one of the two symmetries, it is a very natural question to ask whether there are gapless states that preserve both symmetries. An obvious candidate for such a gapless state is the symmetry-breaking continuous transition. At the transition, the two-point correlation function of the Ising order parameter decays algebraically with the distance, implying that the $\mathbb{Z}_{2}$ zero-form symmetry is indeed unbroken. For the dual $(D-1)$-form symmetry, the 
Kramers-Wannier duality maps the disorder operator, which is a string operator in the Ising basis, to the two-point correlator of the Ising order parameter. Therefore, the expectation value of the disorder operator also exhibits power-law correlation, and the dual zero-form symmetry is preserved. Therefore, the Ising conformal field theory in $(1+1) d$ indeed provides an example of a symmetric gapless state with noninvertible anomaly [31]. But for the case of $D>1$, the situation is far from clear and that is what we will address in this paper. First we analyze the expectation value of the disorder operator in a free field theory.

\section{B. Scaling of disorder operator in field theory}

We now discuss the scaling form of the disorder operator at or near the critical point from a field-theoretical point of view. The natural starting point is the Gaussian fixed point, i.e., a free scalar theory, described by the following Hamiltonian:

$$
H[\phi]=\int d^{D} \mathbf{r}\left[\frac{\pi^{2}}{2}+\frac{1}{2}(\nabla \phi)^{2}\right] .
$$

The real scalar $\phi$ can be thought of as the coarse-grained Ising order parameter, and $\pi$ is the conjugate momentum of the real scalar $\phi$. The $\mathbb{Z}_{2}$ symmetry acts as $\phi \rightarrow-\phi$. The disorder operator $X_{M}$ is basically defined as the continuum version of Eq. (8), where the $\mathbb{Z}_{2}$ symmetry is applied to a finite region $M$.

Interestingly, for the free theory the expectation value of the disorder operator can be related to another well-studied quantity, the second Rényi entanglement entropy $S_{2}$. More precisely, for a region $M$, we have

$$
e^{-S_{2}(M)}=\left\langle X_{M}\right\rangle \text {. }
$$

Here $S_{2}(M)$ is the second Rényi entropy of the region $M$.

To see why this is the case, recall that the second Rényi entropy $S_{2}$ for a region $M$ of a quantum state $|\Psi\rangle$ is given by

$$
e^{-S_{2}(M)}=\operatorname{Tr} \rho_{M}^{2},
$$

where $\rho_{M}$ is the reduced density matrix for the region $M$, obtained from tracing out the degrees of freedom in the complement $\bar{M}: \rho_{M}=\operatorname{Tr}_{\bar{M}}|\Psi\rangle\langle\Psi|$. In the following we denote the ground wave functional of the state $|\Psi\rangle$ by $\Psi(\phi)$ :

$$
|\Psi\rangle=\int D \phi \Psi(\phi)|\phi\rangle .
$$

The Rényi entropy can be calculated with a replica trick, which we now review in the Hamiltonian formalism. Consider two identical copies of the system, in the state $|\Psi\rangle \otimes|\Psi\rangle$. In the field theory example, the fields in the two copies are denoted by $\phi^{(1)}$ and $\phi^{(2)}$, respectively. We denote the basis state with a given field configuration $\phi^{(i)}$ in the $i$ th copy by $\left|\phi_{M}^{(i)}, \phi_{\bar{M}}^{(i)}\right\rangle$, where $\phi_{M}^{(i)}$ is the field configuration restricted to $M$ and similarly $\phi_{\bar{M}}^{(i)}$ for the complement of $M$. Since the two copies are completely identical, there is a swap symmetry $R$ acting between the two copies, $R: \phi^{(1)} \leftrightarrow \phi^{(2)}$. $R_{M}$ then swaps the field configurations only within the region $M$ :

$$
R_{M}\left|\phi_{M}^{(1)}, \phi_{\bar{M}}^{(1)}\right\rangle \otimes\left|\phi_{M}^{(2)}, \phi_{\bar{M}}^{(2)}\right\rangle=\left|\phi_{M}^{(2)}, \phi_{\bar{M}}^{(1)}\right\rangle \otimes\left|\phi_{M}^{(1)}, \phi_{\bar{M}}^{(2)}\right\rangle .
$$

The expectation of $R_{M}$ on the replicated ground state $|\Psi\rangle \otimes$ $|\Psi\rangle$ is then given by

$$
\begin{aligned}
\left(\left\langle\Psi|\otimes\langle\Psi|) R_{M}(|\Psi\rangle \otimes|\Psi\rangle)\right.\right. \\
=\int \prod_{i=1,2} D \phi_{M}^{(i)} D \phi_{\bar{M}}^{(i)} \Psi\left(\phi_{M}^{(1)}, \phi_{\bar{M}}^{(1)}\right) \Psi^{*}\left(\phi_{M}^{(2)}, \phi_{\bar{M}}^{(1)}\right) \\
\quad \times \Psi\left(\phi_{M}^{(2)}, \phi_{\bar{M}}^{(2)}\right) \Psi^{*}\left(\phi_{M}^{(1)}, \phi_{\bar{M}}^{(2)}\right) \\
=\int D \phi_{M}^{(1)} D \phi_{M}^{(2)} \rho_{M}\left(\phi_{M}^{(1)}, \phi_{M}^{(2)}\right) \rho_{M}\left(\phi_{M}^{(2)}, \phi_{M}^{(1)}\right) \\
=\operatorname{Tr} \rho_{M}^{2} .
\end{aligned}
$$

Therefore, the Rényi entropy is the expectation value of the disorder operator for the replica symmetry.

For a free theory, we rotate the basis to $\phi_{ \pm}=\frac{1}{\sqrt{2}}\left(\phi^{(1)} \pm\right.$ $\left.\phi^{(2)}\right)$. In the new basis, the swap symmetry operator becomes

$$
R: \phi_{ \pm} \rightarrow \pm \phi_{ \pm} .
$$

It is straightforward to check that the Hamiltonian of the replica takes essentially the same form in the new basis:

$$
H\left[\phi^{(1)}\right]+H\left[\phi^{(2)}\right]=H\left[\phi_{+}\right]+H\left[\phi_{-}\right] .
$$

The ground state again is factorized: $|\Psi\rangle \otimes|\Psi\rangle=|\Psi\rangle_{+} \otimes$ $|\Psi\rangle_{-}$, where $|\Psi\rangle_{ \pm}$is the state of the $\phi_{ \pm}$field, with the same wave functional as $\phi,\left\langle\phi_{ \pm} \mid \Psi\right\rangle_{ \pm}=\Psi\left(\phi_{ \pm}\right)$as defined in Eq. (14).

We can now compute the expectation value of $R_{M}$ :

$$
\left(\left\langle\left.\Psi\right|_{+} \otimes\left\langle\left.\Psi\right|_{-}\right) R_{M}\left(|\Psi\rangle_{+} \otimes|\Psi\rangle_{-}\right)=\left\langle X_{M}\right\rangle,\right.\right.
$$

where we used the fact that $R$ acts as the identity on $\phi_{+}$. For $\phi_{-}, R_{M}$ is nothing but the disorder operator $X_{M}$.

The second Rényi entropy of a free scalar has been well studied [32-38] and we summarize the results below.

It is important to distinguish the case where the boundary is smooth and the case with sharp corners on the boundary.

First consider a smooth boundary. For a sphere of radius $R$, in $D=1,2,3$ we have

$$
S_{2}= \begin{cases}\frac{1}{6} \ln R, & D=1 \\ a_{1} \frac{R}{\epsilon}-\gamma, & D=2 \\ a_{2}\left(\frac{R}{\epsilon}\right)^{2}-\frac{1}{192} \ln \frac{R}{\epsilon}, & D=3 .\end{cases}
$$

Here $\epsilon$ is a short-distance cutoff, e.g., the lattice spacing, $a_{1}, a_{2}$ nonuniversal coefficients, and $\gamma$ a universal constant. For a more general smooth entangling boundary, in two dimensions the same form holds although the constant correction $\gamma$ depends on shape of the region. In three dimensions, it is known that the coefficient of the logarithmic divergent part of the Rényi entropy can be determined entirely from the local geometric data (e.g., curvature) of the surface in a general CFT $[39,40]$.

If the boundary has sharp corners then there are additional divergent terms in the entropy. The prototypical case is $D=2$ when the entangling region has sharp corners. In that case

$$
S_{2}=a_{1} \frac{l}{\epsilon}-s \ln \frac{l}{\epsilon},
$$

where $l$ is the perimeter of the entangling region and $s$ is a universal function that only depends on the opening angles 
(a)

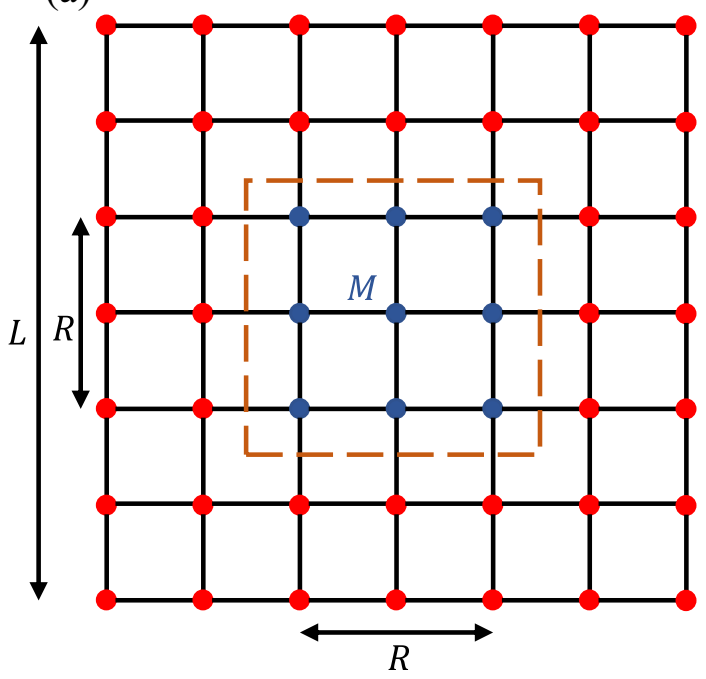

(b)

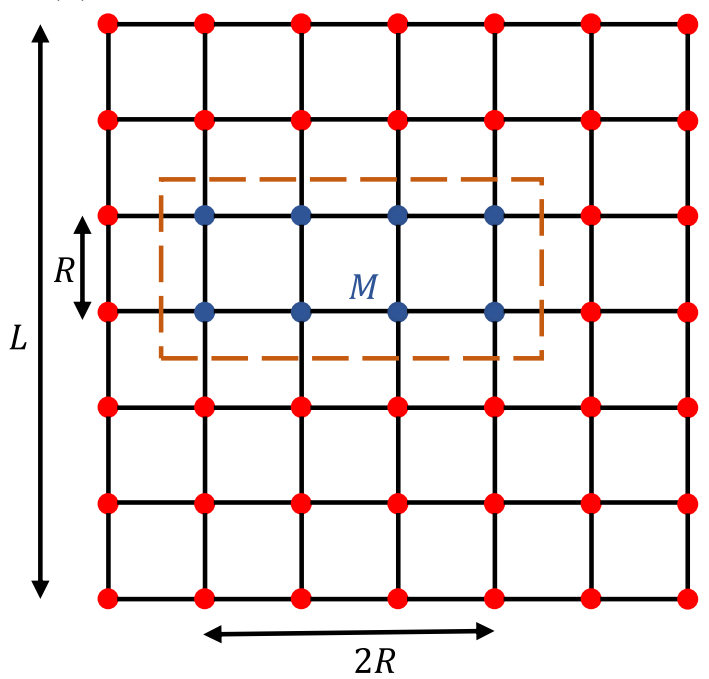

FIG. 3. Disorder operator $X$ applied on regions with different shapes: (a) $M$ is a square region with size $R \times R$ and perimeter $l$. (b) $M$ is a rectangular region with size $R \times 2 R$.

of the corners. For a real free scalar, the coefficient of the logarithmic correction is $s \approx 0.0260$ for a square region (so four $\pi / 2$ corners, as those in Fig. 3) [33,36].

Qualitatively, it is important that for $D=2,3$ the leading term in $S_{2}$ always obeys a "perimeter" law; i.e., it only depends on the "area" (length in two dimensions) of the entangling boundary. If instead we view $S_{2}$ as the disorder operator for the $\mathbb{Z}_{2}$ replica symmetry, the nonuniversal, cutoff-dependent perimeter term can be removed by redefining the disorder operator locally along the boundary, and the remaining term is universal. For $D=2$, the subleading term is either a negative constant when the boundary is smooth, or a $\ln l$ correction with a negative coefficient. So according to Eq. (12), the disorder parameter $\left\langle X_{M}\right\rangle$, after renormalizing away the perimeter term, does not decrease with the size of $M$, and therefore the corresponding $(D-1)$-form symmetry is spontaneously broken. This is consistent with the fact that the replica symmetry itself must be preserved as there is no coupling between the two copies.

Although the free Gaussian theory is unstable against quartic interactions below the upper critical dimension, and the actual critical theory is the interacting Wilson-Fisher fixed point, results from the free theory can still provide useful insights. It is well known that for $D=1$, for $M$ an interval of length $R$, the disorder operator $\left\langle X_{M}\right\rangle \sim R^{-1 / 4}$, the same power-law decay as that of the Ising order parameter due to Kramers-Wannier duality. For $D=2$, we will resort to numerical simulations below to address the question.

Notice that the relation between $\langle X\rangle$ and $S_{2}$ essentially holds for all free theories, including free fermions. For example, the disorder operator associated with the fermion parity symmetry is also equal to $S_{2}$. Interestingly, for a Fermi liquid, it is well known that $\ln \langle X\rangle=-S_{2} \sim-l^{D-1} \ln l$ [41,42], where here $l$ is the linear size of the region. This is an example of a gapless state where the $(D-1)$-form symmetry is preserved. Similar results hold for noninteracting bosonic systems with "Bose surface" [43], an example of which in two dimensions is given by the exciton Bose liquid [44,45]:

$$
H=\int d^{2} \mathbf{r}\left[\frac{\pi^{2}}{2}+\kappa\left(\partial_{x} \partial_{y} \phi\right)^{2}\right] .
$$

In other words, to preserve both the zero-form symmetry and the dual $(D-1)$-form symmetry, it is necessary to have a surface of gapless modes in the momentum space.

While analytical results discussed in this work are limited to free theories, we conjecture that similar scaling relations hold for interacting CFTs as well. To see why this is plausible, we notice that the entanglement Hamiltonian of a CFT is algebraically "localized" near the boundary of the subsystem [46], which suggests that even for a nonlocal observable, such as the disorder operator, the major contribution is expected to come from the boundary, and hence a perimeter law scaling. We leave a more systematic study along these lines for future work. In Sec. IV we numerically confirm our conjecture for the Ising CFT in $(2+1) d$.

We now briefly discuss what happens if a small mass is turned on in Eq. (11). Suppose we are in a gapped phase, and denote by $\xi$ the correlation length. In general, we expect that $S_{2}$ obeys a perimeter scaling in the gapped phase; namely, the leading term in $S_{2}$ is given by $a \frac{R}{\epsilon}$. In two dimensions for a disk entangling region of radius $R$, we have [47]

$$
S_{2}=a_{c} \frac{R}{\xi}+f\left(\frac{R}{\xi}\right) \text {. }
$$

Here $a_{c}$ is the value of $a$ at the critical point [which was denoted by $a_{1}$ in Eq. (20)]. The function $f(x)$ satisfies

$$
f(x) \rightarrow \begin{cases}r x, & x \rightarrow \infty \\ -\gamma_{c}, & x \rightarrow 0 .\end{cases}
$$

Here $r$ is a universal constant (once the definition of $\xi$ is fixed). Suppose the transition is tuned by an external parameter $g$ and the critical point is reached at $g_{c}$. Since $\xi \sim$ $\left(g-g_{c}\right)^{-v}$ where $v$ is the correlation length exponent, one finds that

$$
a-a_{c} \sim\left(g-g_{c}\right)^{\nu}
$$




\section{ORDER AND DISORDER IN ISING SPIN MODELS}

In the following we study 1 -form symmetry breaking in the transverse field Ising (TFI) model which gives rise to the $(2+1) d$ Ising transition. We have reviewed the connection with the $\mathbb{Z}_{2}$ gauge theory in Sec. II, as well as the 1 -form symmetry in the Ising spin system. We will now focus more on the quantitative aspects of the TFI model. Even though the TFI model and the $\mathbb{Z}_{2}$ lattice gauge theory are equivalent by the duality map, we choose to work with the TFI model here because the numerical simulation is more straightforward.

We will now consider a square lattice with one Ising spin per site, and the global Ising symmetry is generated by $S=\prod_{\mathbf{r}} \sigma_{\mathbf{r}}^{x}$. There are, generally speaking, two phases: a "disordered" phase, where the Ising symmetry is preserved by the ground state, ${ }^{2}$ and an ordered phase, where the ground states spontaneously break the symmetry. They are separated by a quantum phase transition, described by a conformal field theory with $\mathbb{Z}_{2}$ symmetry. It is well understood how to characterize the Ising symmetry breaking (and its absence) in the three cases: consider the two-point correlation function of the order parameter $\sigma_{\mathbf{r}}^{z}$. The asymptotic forms of the correlation function $\left\langle\sigma_{\mathbf{r}}^{z} \sigma_{\mathbf{r}^{\prime}}^{z}\right\rangle$ for large $\left|\mathbf{r}-\mathbf{r}^{\prime}\right|$ distinguish the three cases:

$$
\left\langle\sigma_{\mathbf{r}}^{z} \sigma_{\mathbf{r}^{\prime}}^{z}\right\rangle \sim \begin{cases}e^{-\frac{\left|r-\mathbf{r}^{\prime}\right|}{\xi}} & \text { (disordered) } \\ \frac{1}{\left|\mathbf{r}-\mathbf{r}^{\prime}\right|^{2 \Delta}} & \text { (critical) } \\ \text { const } & \text { (ordered). }\end{cases}
$$

In both the disordered phase and the quantum critical point, the Ising symmetry is preserved because of the absence of long-range order. The prototypical lattice model that displays all these features is the TFI model defined on a square lattice:

$$
H=-\sum_{\left\langle\mathbf{r r}^{\prime}\right\rangle} \sigma_{\mathbf{r}}^{z} \sigma_{\mathbf{r}^{\prime}}^{z}-h \sum_{\mathbf{r}} \sigma_{\mathbf{r}}^{x}, h \geqslant 0 .
$$

Note that this is the same as Eq. (9), but we have set $J=1$ and renamed $K$ by $h$, to align with the standard convention in the literature. The model is in the ordered (disordered) phase for $h \ll 1(h \gg 1)$. The precise location of the critical point varies with dimension, $h_{c}=1$ in $D=1$ and $h_{c}=3.044$ in $D=2$ [48,49].

We will be interested in the disorder operator:

$$
X_{M}=\prod_{\mathbf{r} \in M} \sigma_{\mathbf{r}}^{x}
$$

where $M$ is a rectangle region in the lattice, illustrated in Fig. 3. In Ref. [14] this operator is called the patch symmetry operator.

When $X_{M}$ is applied to, e.g., $|\uparrow \cdots \uparrow\rangle$, a domain wall is created along the boundary of the region $M$. These operators are charged under the dual $\mathbb{Z}_{2} 1$-form symmetry. One can easily see that $\left\langle\psi_{h=\infty}\left|X_{M}\right| \psi_{h=\infty}\right\rangle=1$, and $\left\langle\psi_{h=0}\left|X_{M}\right| \psi_{h=0}\right\rangle=0$. More generally,

$$
\left\langle\psi\left|X_{M}\right| \psi\right\rangle \sim \begin{cases}e^{-a l_{M}}, & h>h_{c} \\ e^{-b A_{M}}, & h<h_{c},\end{cases}
$$

\footnotetext{
${ }^{2}$ We note that there are in fact two distinct types of Ising-disordered phases in $2 \mathrm{D}$, one trivial paramagnet and the other one a nontrivial Ising symmetry-protected topological phase.
}

when $M$ is sufficiently large compared to the correlation length. Here $l$ is the perimeter of the boundary of $M$, and $A$ is the area of $M$. The coefficients $a$ and $b$ can be computed perturbatively in the limit of large and small $h$. In two dimensions, take $M$ to be a square of perimeter $l$, so $\operatorname{Perimeter}(M)=l$ and the $\operatorname{Area}(M)=l^{2} / 16$. We can find that for large $l$

$$
-\ln \langle X\rangle= \begin{cases}\frac{l}{8 h^{2}}, & h \gg h_{c} \\ \frac{1}{4}|\ln h| l^{2}, & h \ll h_{c} .\end{cases}
$$

\section{NUMERICAL SIMULATIONS}

In this section we study the disorder operator in the $(2+$ 1)d TFI model. We employ the stochastic series expansion (SSE) quantum Monte Carlo method [50-53] to simulate the Hamiltonian in Eq. (27). In particular, to be able to directly access the disorder operator in Eq. (28), instead of implementing the algorithm in the conventional $\sigma^{z}$ basis we choose to work in the $\sigma^{x}$ basis and construct the highly efficient directed loop algorithm therein [51]. The implementation details of the SSE-QMC algorithm are given in the Appendix.

In our numerical simulations, we choose $M$ to be a rectangular region of size $R_{1} \times R_{2}$ (i.e., the region contains $R_{1} R_{2}$ sites), and denote the perimeter $l=2\left(R_{1}+R_{2}\right)$. As shown in Figs. 3(a) and 3(b), for finite-size studies, we fix the aspect ratio $R_{2} / R_{1}=1$ of square shape and 2 of rectangle shape. The linear system size of the lattice is $L$ and at the critical point we scale the inverse temperature $\beta=1 / T \sim L$ to access the thermodynamic limit.

\section{A. Disordered phase $h>h_{c}$}

First we present results in the disordered phase $h>h_{c}$. As shown in Eq. (29), we expect that the disorder operator obeys a perimeter law scaling, and for $h \gg h_{c}$ the coefficient is given in Eq. (30).

Figure 4 shows the QMC-obtained $\ln \left\langle X_{M}\right\rangle$ as a function of $l$ for different values of $h$. The temperature is taken to be $\beta=$ 10 , and we have checked that the results already converge for this value of $\beta$. We observe a clear linear scaling, and the inset shows that for large field $h \gg h_{c}$, the slopes of the $\ln \left\langle X_{M}\right\rangle$ are indeed given by $1 / 8 h^{2}$ asymptotically.

Now we consider the other limit, when $h$ is approaching the critical point $h_{c}$ from the disordered side. To test the scaling given in Eq. (25), we measure the disorder operator and find the slope $a$ by a linear fit. Figure 5 shows $a_{c}-a$ as a function of $h-h_{c}$ in a log-log plot. A clear power law manifests in the data, and the exponent is found to be $v=0.63(2)$. Considering the finite-size effect, the result agrees very well with the three-dimensional (3D) Ising correlation length exponent.

\section{B. Critical point $h=h_{c}$}

The central question to be addressed is whether the $\mathbb{Z}_{2} 1$ form symmetry is spontaneously broken at the critical point. To this end, we measure the disorder operator $\langle X\rangle$ at $h=h_{c}$ and scale the inverse temperature $\beta=L$ in these simulations. We have also checked that the finite- $\beta$ effect is negligible in our calculations.

Figure 6 shows $\ln \left\langle X_{M}\right\rangle$ as a function of the perimeter $l$, where $M$ is taken to be a square region, as illustrated in 


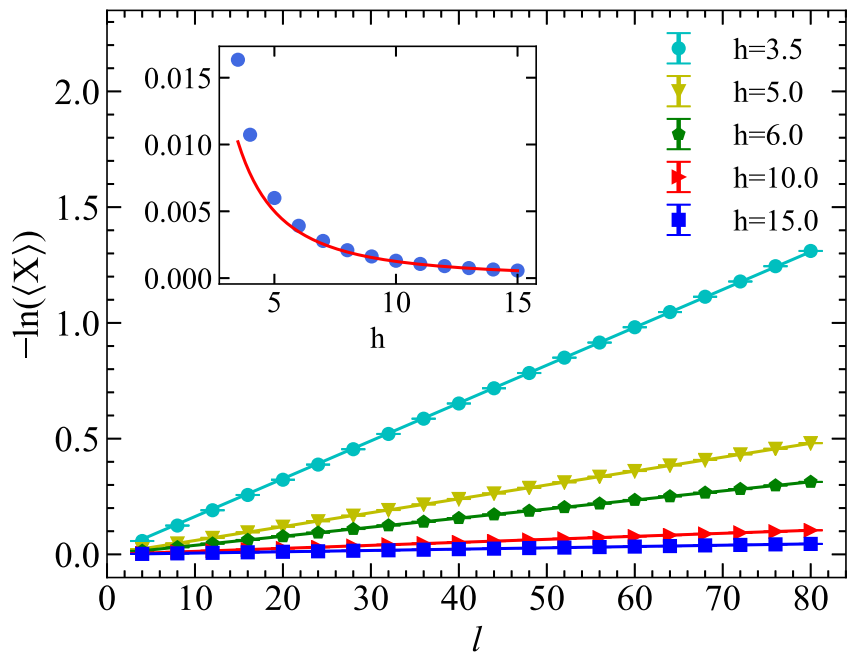

FIG. 4. $-\ln (\langle X\rangle)$ versus $l$ at $L=32$ for different $h$ in disordered phases. We use the straight line to fit the data of different external fields and put the obtained slopes in the inset. One sees that as $h \gg$ $h_{c}$, the fitted slopes (blue circles) approach the predicted relation $y=$ $\frac{1}{8 h^{2}}$ (red line). The fitting errors are negligible compared to the circle size.

Fig. 3(a). Results for different system sizes $L=8,16,24$, 32 , and 40 are presented and it is clear that the finite-size effect is negligible. The data clearly demonstrate a linear scaling as in Eq. (21) and the slope $a_{1}$ quickly converges to $0.0394 \pm 0.0004$.

As we have explained, the boundary of $M$ generally contributes to the disorder operator a term proportional to the perimeter. To detect 1-form symmetry breaking, we need to check whether $\langle X\rangle$ depends on the area or not. For this purpose, we consider rectangular regions with different aspect ratios: one with 1:1 [Fig. 3(a)] and the other with 1:2 [Fig. 3(b)], and present the results of $\langle X\rangle$ at the $h=h_{c}$ together in Fig. 7. It can be seen that the two sets of data

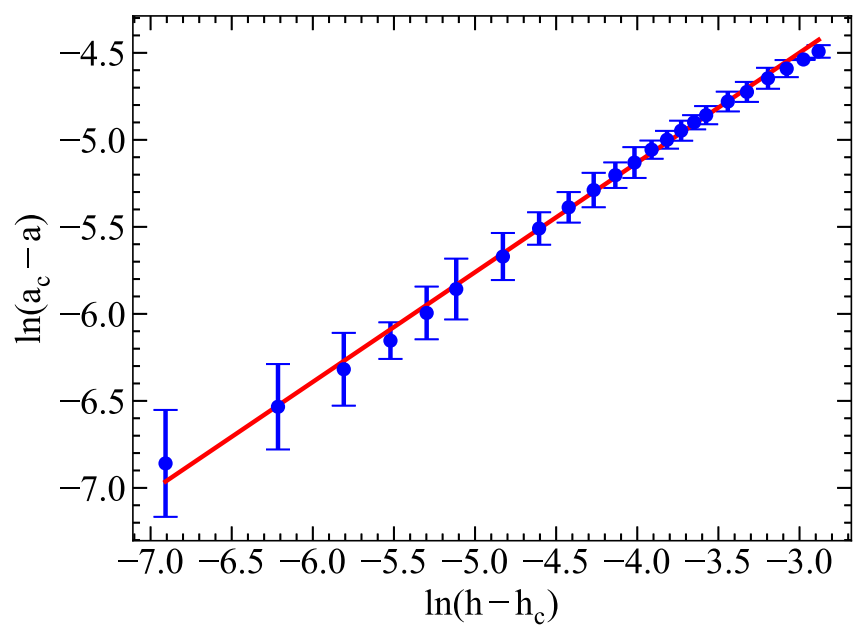

FIG. 5. $\ln \left(a_{c}-a\right)$ versus $\ln \left(h-h_{c}\right)$ in the disordered phase for $L=24$ when $h$ is approaching the critical point. The fitted slope (red line) is $0.63 \pm 0.02$, consistent with the correlation length exponent of the $(2+1) d$ Ising transition, as expected in Eq. (25).

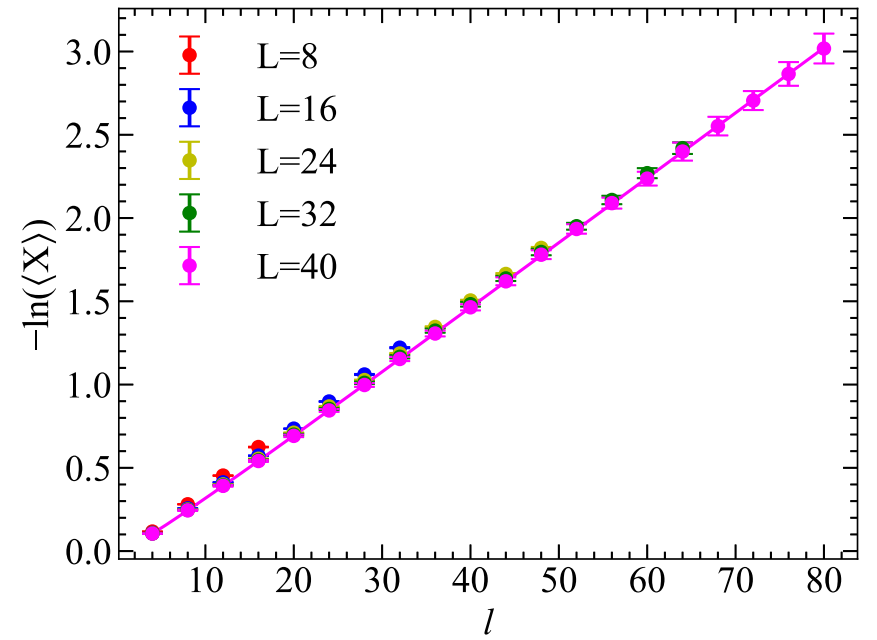

FIG. 6. $-\ln (\langle X\rangle)$ versus $l$ at the critical point. We use the relation of Eq. (21) to fit the data and the fitted curve of the data up to $L=40$ is $-\ln (\langle X\rangle)=(0.0394 \pm 0.0004) l-(0.0267 \pm$ $0.005) \ln (l)-(0.0158 \pm 0.008)$.

basically fall on the same curve, indicating that the disorder parameter only depends on the perimeter.

Given the relation between $\left\langle X_{M}\right\rangle$ and the Rényi entropy in the free theory, let us examine possible corner contributions to $\left\langle X_{M}\right\rangle$, which is parametrized in the coefficient $s$ of Eq. (21). We fit the data points in Fig. 7 to Eq. (21), which yields $s=0.0272 \pm 0.004$, close to the free value. We perform the same fit for data points with aspect ratio 1:2 and obtain essentially the same results $(s=0.0279 \pm 0.003)$. The agreement between the fitting results for regions with different aspect

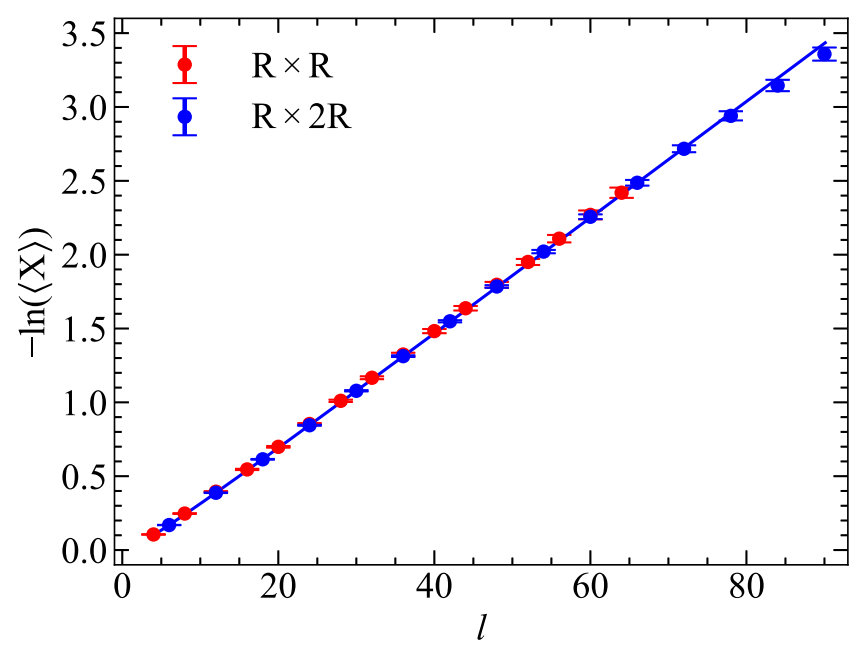

FIG. 7. $-\ln \left\langle X_{M}\right\rangle$ versus $l$ at the phase transition point for $M$ with the shape $R \times R$ (already shown in Fig. 6) and $R \times 2 R$, for system size $L=32$. The blue line represents the fitted curve of the data for $R \times 2 R$ using the relation specified in Eq. (21). The fitted result of $R \times 2 R$ is $-\ln \langle X\rangle=(0.0397 \pm 0.0002) l-(0.0279 \pm$ $0.003) \ln (l)-(0.0192 \pm 0.006)$ and for $R \times R$ at $L=32$ the result is $-\ln \langle X\rangle=(0.0399 \pm 0.0003) l-(0.0272 \pm 0.004) \ln (l)-$ $(0.0162 \pm 0.005)$. The coefficients are indistinguishable within error bars. 


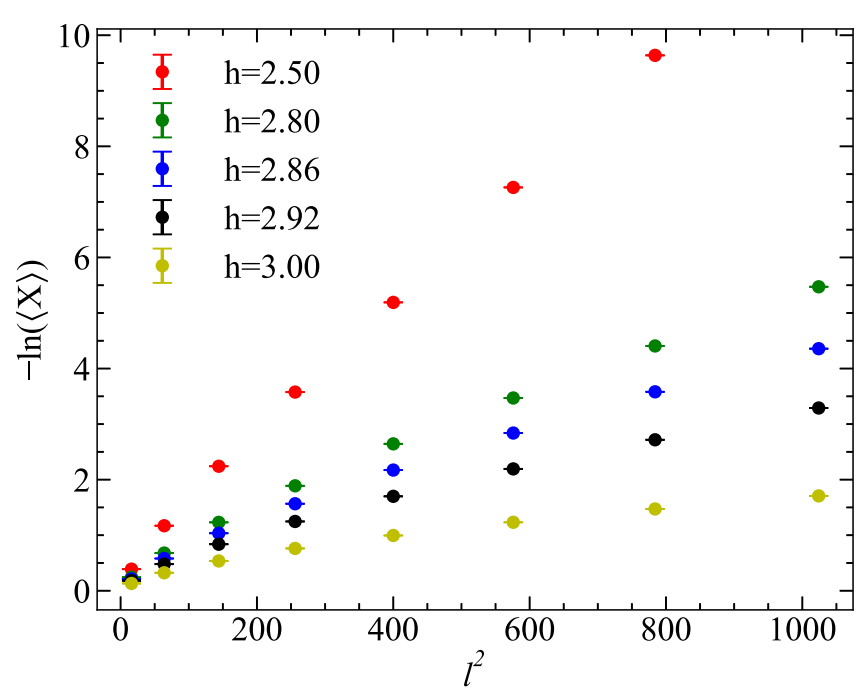

FIG. 8. $-\ln \langle X\rangle$ versus $l^{2}$ when $L=16$ and $\beta=32$. The transverse fields are chosen from inside the ordered phase $(h=2.5)$ to near the critical point $(h=3.0)$. The area law scaling in the disorder operator clearly manifests, and the slope increases as one moves deeper in the ferromagnetic phase.

ratios again lends strong support for the perimeter dependence of $\left\langle X_{M}\right\rangle$ even beyond the leading order, and consequently the 1 -form symmetry breaking at the $(2+1) d$ Ising CFT.

The convergence of the coefficients $a_{1}, s$, and $a_{0}$ versus the linear system size $L$ is given in Fig. 9 in Appendix A 3.

\section{Ordered phase $h<\boldsymbol{h}_{c}$}

For $h<h_{c}$ where Ising spins order ferromagnetically, our algorithm becomes inefficient because we choose to work in the $\sigma^{x}$ basis to facilitate the computation of the disorder operator. Nevertheless, simulations indeed find that the disorder parameter decays much more rapidly with the linear size of the region, consistent with the area law in Eqs. (5) and (29). $-\ln \left\langle X_{M}\right\rangle$ as a function of $l^{2}$ is shown in Fig. 8 for different values of $h$ below the critical value. It is clear that as we go deep into the ordered phase, the slope $b$ increases as expected and the data points converge to a straight line for large $l^{2}$. For $h=3.0$ very close to the critical point, one can observe that for relatively small values of $l^{2}$ the data points do not scale linearly, which can be attributed to a subleading perimeter dependence.

\section{CONCLUSION AND DISCUSSION}

As discussed in the beginning of the paper, in recent years, new types of quantum phases and phase transitions that are "beyond Laudau" are flourishing, exhibiting topological order, emergent gauge field, and fractionalization. Higher-form symmetries and their spontaneous breaking are new conceptual tools introduced to provide a unified framework for both conventional and exotic phases. A quantum phase, gapped or gapless, is fundamentally characterized by its emergent symmetry and the associated anomaly. While the philosophy went back to the Landau classification of phase transitions, the power of this perspective has only begun to unfold recently with the introduction of generalized global symmetries.

Here we reexamine the familiar Ising symmetry-breaking transition, arguably the simplest conformal field theory, from the emergent symmetry perspective. A $D$-dimensional Ising system has a "hidden" $\mathbb{Z}_{2}(D-1)$-form symmetry, whose charges are Ising domain walls. Gapped phases in this system are associated to the spontaneous breaking of the enlarged symmetry [zero-form and $(D-1)$-form symmetries]. It is then of great interest to determine the symmetry-breaking pattern at the critical point, to complete our understanding of the global phase diagram from the emergent symmetry point of view.

In this work we determine the scaling form of the disorder operator in Ising CFTs when $D>1$. The most challenging case is $D=2$ where the transition is described by the interacting Wilson-Fisher fixed point, and we exploit large-scale quantum Monte Carlo simulations. We use the disorder operator of the Ising system to probe the breaking of the dual higher-form symmetry. We find numerically that at the critical point of the two-dimensional (2D) quantum Ising model the one-form disorder operator exhibits spontaneous symmetry breaking as in the disordered phase, whereas in the ordered phase, the 1-form symmetry is intact.

The disorder operator is intimately related to a line defect (also called a twist operator) in an Ising CFT, around which the spin operator sees an antiperiodic boundary condition. In fact, a line defect is nothing but the boundary of a disorder operator. It is believed that in general such a line defect can flow to a conformal one at low energy, which is indeed consistent with a perimeter law scaling for the expectation value of the disorder operator. ${ }^{3}$ Local properties of disorder line defects have been previously investigated in Refs. [54,55]. It will be interesting to understand the relation between the local properties with the universal corner contributions to the disorder operator [56].

Our findings, besides elucidating the physics of quantum Ising systems from a different angle, provide a working example of higher-form symmetry at practical use. Similar physical systems can be studied; for example, the disordered operator constructed in this work is readily generalized to the $(2+1) d$ $\mathrm{XY}$ transition and can be measured with unbiased QMC simulations. Another important direction is to study other higher-form symmetry-breaking transitions, such as 1-form symmetry-breaking transition in $3 \mathrm{D}$ systems. It would also be interesting to investigate the utility of the disorder operator in the topological Ising paramagnetic phase. More applications in quantum lattice models are awaiting to be explored, and will certainly lead to new insight for a new framework that unifies our understanding of the exotic quantum phases and transitions going beyond the Landau paradigm and those within.

Note added. We would like to draw the reader's attention to few closely related recent works by Wu, Jian, and $\mathrm{Xu}[20,22]$ and by some of the present authors on scaling of the disorder operator at $(2+1) d \mathrm{U}(1)$ quantum criticality [21].

\footnotetext{
${ }^{3}$ We are grateful for Shu-Heng Shao for discussions on this point.
} 


\section{ACKNOWLEDGMENTS}

J.R.Z., Z.Y., and Z.Y.M. thank the enjoyable discussions with Yan-cheng Wang and Yang Qi and acknowledge the support from the RGC of Hong Kong SAR of China (Grants No. 17303019 and No. 17301420), MOST through the National Key Research and Development Program (Grant No. 2016YFA0300502), and the Strategic Priority Research Program of the Chinese Academy of Sciences (Grant No. XDB33000000). We are grateful for Xiao-Gang Wen, ShuHeng Shao, and William William-Krempa for helpful comments. M.C. would like to thank Zhen $\mathrm{Bi}$, Wenjie Ji, and Chao-Ming Jian for enlightening discussions and acknowledges support from NSF (Grant No. DMR-1846109) and the Alfred P. Sloan Foundation. We thank the Computational Initiative at the Faculty of Science and the Information Technology Services at the University of Hong Kong, and the Tianhe-1A, Tianhe-2, and Tianhe-3 prototype platforms at the National Supercomputer Centers in Tianjin and Guangzhou for their technical support and generous allocation of CPU time. We acknowledge Beijng PARATERA Tech CO., Ltd. for providing HPC resources that have contributed to the research results reported within this paper.

\section{APPENDIX: QUANTUM MONTE CARLO IMPLEMENTATION OF DISORDER OPERATOR}

In this Appendix, we describe the implementation of the SSE-QMC algorithm of the quantum Ising model, in particular the implementation of the disorder operator which involves a change of basis.

\section{SSE on $\sigma^{z}$ basis}

The Hamiltonian for the transverse field Ising model is

$$
H=-\sum_{\left\langle\mathbf{r} \mathbf{r}^{\prime}\right\rangle} \sigma_{\mathbf{r}}^{z} \sigma_{\mathbf{r}^{\prime}}^{z}-h \sum_{\mathbf{r}} \sigma_{\mathbf{r}}^{x} .
$$

Then we can decompose the Hamiltonian into site and bond operators

$$
\begin{aligned}
H_{0,0} & =I, \\
H_{-1, a} & =h\left(\sigma_{a}^{+}+\sigma_{a}^{-}\right), \\
H_{0, a} & =h, \\
H_{1, a} & =\left(\sigma_{\mathbf{r}(a)}^{z} \sigma_{\mathbf{r}^{\prime}(a)}^{z}+1\right),
\end{aligned}
$$

with $H=-\sum_{i=-1}^{1} \sum_{a} H_{i, a}$. Here $H_{0,0}$ denotes the identity operator and $i=-1,0,1$ indicates different types of operator: off-diagonal operator on site, diagonal operator on site, and diagonal operator on bond. The subscript $a$ holds two different identities: for bond operators $H_{1, a}$ the index $a$ denotes the bond number (e.g., for the 2D case $a=1,2, \ldots, N_{b}=2 L^{2}$ ); and for site operators $H_{0, a}$ and $H_{-1, a}$ the index $a$ denotes the site number (e.g., for the $2 \mathrm{D}$ case $a=1,2, \ldots, N=L^{2}$ ).

Next, the partition function $Z=\operatorname{Tr} e^{-\beta H}$ can be expressed as a power series expansion:

$$
Z=\sum_{\alpha} \sum_{S_{M}} \frac{\beta^{n}(M-n) !}{M !}\left\langle\alpha\left|\prod_{i=1}^{M} H_{a_{i}, p_{i}}\right| \alpha\right\rangle,
$$

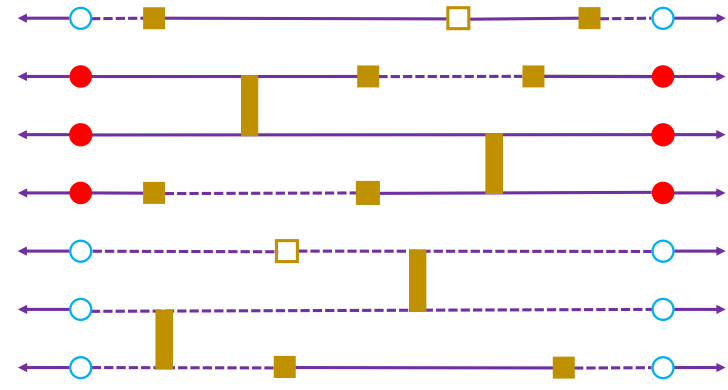

FIG. 9. SSE-QMC configuration of the quantum Ising model. Golden bars represent Ising bond operators. Solid square plaquettes are off-diagonal site operators, and open plaquettes denote the diagonal site operators. Arrows represent periodic boundary conditions in the imaginary time direction. The red solid circles and the light blue open circles indicate spin up and down. Solid and dashed purple lines illustrate the spin states (spin up or down).

where $M$ is the truncation of the expansion series $n$. Taking $\sigma^{z}$ as a complete set of basis for the system, the nonzero matrix elements for site operators and bond operators are

$$
\begin{aligned}
\left\langle\uparrow\left|H_{-1, a}\right| \downarrow\right\rangle & =\left\langle\downarrow\left|H_{-1, a}\right| \uparrow\right\rangle=h, \\
\left\langle\uparrow\left|H_{0, a}\right| \uparrow\right\rangle & =\left\langle\downarrow\left|H_{0, a}\right| \downarrow\right\rangle=h, \\
\left\langle\uparrow \uparrow\left|H_{1, a}\right| \uparrow \uparrow\right\rangle & =\left\langle\downarrow \downarrow\left|H_{1, a}\right| \downarrow \downarrow\right\rangle=2 .
\end{aligned}
$$

The updating scheme [50] includes the diagonal update which either inserts or removes a diagonal operator between two states with probabilities regulated by the detailed balanced condition, and the cluster update which flips all the spins on the cluster with the Swendsen-Wang scheme. The configurations of the updating scheme are shown in Fig. 9.

We describe the updating scheme in the following steps.

(1) Diagonal update: We go through the operator strings and either remove or insert a diagonal operator according to the following procedures.

(a) For a diagonal operator $\left(H_{0, a}\right.$ or $\left.H_{1, a}\right)$, we removed it with probability

$$
P=\min \left(\frac{M-n+1}{\beta\left(h N+2 N_{b}\right)}, 1\right),
$$

where $N$ denotes the number of lattice sites, and $N_{b}$ denotes the number of bonds.

(b) For a null operator $\left(H_{0,0}\right)$, we substitute it with a diagonal operator $H_{1, a}$ or $H_{0, a}$ by the procedures below.

(i) First we make the decision of which kind of diagonal operators to insert. We choose the type of $H_{1, a}$ with probability

$$
P(h)=\frac{2 N_{b}}{h N+2 N_{b}}
$$

or the type $H_{0, a}$ with probability $1-P(h)$.

(ii) After the decision is made, we accept the insertion of an operator with probability

$$
P=\min \left(\frac{\beta\left(h N+2 N_{b}\right)}{M-n}, 1\right),
$$

and after that we choose a random and appropriate site or bond to insert the operator. If the chosen bond to 
insert a bond operator has an antiparallel configuration, then the insertion of a bond operator at this place is prohibited.

(c) For an off-diagonal operator, we ignore it and go to the next operator in the operator strings.

(2) Cluster update:

(a) We generally follow two rules to construct the clusters: (1) clusters are terminated on site operators $H_{-1, a}$ or $H_{0, a}$; and (2) the four legs of a bond operator $H_{1, a}$ belong to one cluster. Carry out this procedure until all the clusters are built, and a configuration of clusters is shown in Fig. 9.

(b) Clusters identified from the above rules are then flipped with probability $1 / 2$ (which is the Swendsen-Wang cluster updating scheme).

Since the disorder operator is a product of $\sigma^{x}$, i.e., $\left\langle X_{M}\right\rangle=$ $\left\langle\prod_{\mathbf{r} \in M} \sigma_{\mathbf{r}}^{x}\right\rangle$, it is a measurement of an off-diagonal operator in the $\left\{\sigma^{z}\right\}$ basis. In the $\sigma^{z}$ basis, the off-diagonal operator can be measured if the operator is a product of operators in the Hamiltonian. It is proved in Ref. [51] that

$$
\left\langle\prod_{i=1}^{m} \hat{H}_{k_{i}}\right\rangle=\frac{1}{(-\beta)^{m}}\left\langle\frac{(n-1) !}{(n-m) !} N\left(k_{1}, \ldots, k_{m}\right)\right\rangle_{W},
$$

where $N\left(k_{1}, \ldots, k_{m}\right)$ denotes the number of ordered subsequences $k_{1}, \ldots, k_{m}$ in $S_{n}$. However, this measurement becomes practically impossible when the length of the products becomes sufficiently large, because $\frac{1}{(-\beta)^{m}} \frac{(n-1) !}{(n-m) !}$ would grow to a very large value as $m$ increases; thus $N\left(k_{1}, \ldots, k_{m}\right)$ would be too small to measure within the limited computing power. So the measurement of $\langle X\rangle$ in the $\left\{\sigma^{z}\right\}$ basis seems hopeless. To solve this problem, we need to change the basis to make $\sigma^{x}$ diagonal.

\section{SSE on $\sigma^{x}$ basis}

Since we need to measure the disorder operator which is defined as the nonlocal product of off-diagonal operators, and it is extremely hard to measure it in the traditional $\sigma^{z}$ basis, we then turn to the $\sigma^{x}$ basis as the complete set of basis of the system, and we can use directed loop algorithms [51,52] to simulate this model.

For convenience, we now write the $\sigma^{x(z)}$ above as $\sigma^{z(x)}$ in the following; the Hamiltonian can be rewritten as

$$
H=-\sum_{\left\langle\mathbf{r r}^{\prime}\right\rangle} \sigma_{\mathbf{r}}^{x} \sigma_{\mathbf{r}^{\prime}}^{x}-h \sum_{\mathbf{r}} \sigma_{\mathbf{r}}^{z}+N_{b} \Delta .
$$

Here $\left\langle\mathbf{r r}^{\prime}\right\rangle$ refers to the nearest neighbors. $N_{b}$ is the number of bonds. $N_{b} \Delta$ is a constant added to the Hamiltonian to ensure that the matrix elements defined in Eq. (A14) are positive definite. Rewriting $S^{x}$ with $S^{+}+S^{-}$, we can decompose the Hamiltonian as

$$
H=-\sum_{b=1}^{N_{b}} H_{b}
$$

with

$$
H_{b}=-H_{1, b}-H_{2, b}+H_{3, b} .
$$

Here $b$ refers to the bond number, and $H_{1, b}, H_{2, b}, H_{3, b}$ are defined as follows:

$$
\begin{aligned}
& H_{1, b}=\sigma_{\mathbf{r}(b)}^{+} \sigma_{\mathbf{r}^{\prime}(b)}^{+}+\sigma_{\mathbf{r}(b)}^{-} \sigma_{\mathbf{r}^{\prime}(b)}^{-}, \\
& H_{2, b}=\sigma_{\mathbf{r}(b)}^{+} \sigma_{\mathbf{r}^{\prime}(b)}^{-}+\sigma_{\mathbf{r}(b)}^{-} \sigma_{\mathbf{r}^{\prime}(b)}^{+}, \\
& H_{3, b}=\Delta-a h\left(\sigma_{\mathbf{r}(b)}^{z}+\sigma_{\mathbf{r}^{\prime}(b)}^{z}\right) .
\end{aligned}
$$

Note that $a=\frac{N}{2 N_{b}}$ and $N$ is the number of lattice sites. For the one-dimensional (1D) case $a=\frac{1}{2}$, and for the 2D case $a=\frac{1}{4}$. The nonzero matrix elements for the diagonal operators are

$$
\begin{aligned}
& \left\langle\uparrow \uparrow\left|H_{b}\right| \uparrow \uparrow\right\rangle=\Delta-2 a h, \\
& \left\langle\downarrow \downarrow\left|H_{b}\right| \downarrow \downarrow\right\rangle=\Delta+2 a h, \\
& \left\langle\uparrow \downarrow\left|H_{b}\right| \uparrow \downarrow\right\rangle=\left\langle\downarrow \uparrow\left|H_{b}\right| \downarrow \uparrow\right\rangle=\Delta .
\end{aligned}
$$

In the simulation we set $\Delta=2 a h+1$ to make sure $H_{b}$ is positive definite. The off-diagonal matrix elements are

$$
\begin{aligned}
\left\langle\uparrow \uparrow\left|H_{b}\right| \downarrow \downarrow\right\rangle= & \left\langle\downarrow \downarrow\left|H_{b}\right| \uparrow \uparrow\right\rangle \\
& =\left\langle\uparrow \downarrow\left|H_{b}\right| \downarrow \uparrow\right\rangle=\left\langle\downarrow \uparrow\left|H_{b}\right| \uparrow \downarrow\right\rangle=1 .
\end{aligned}
$$

Then the updating scheme becomes as follows.

(1) Diagonal update: The purpose of the diagonal update which either inserts or removes a diagonal between two basis states is to change the expansion order $n$ by \pm 1 . The corresponding acceptance probability is

$$
\begin{aligned}
P(\text { insert }) & =\frac{N_{b} \beta\left\langle\alpha(p)\left|H_{1, b}\right| \alpha(p)\right\rangle}{M-n}, \\
P(\text { remove }) & =\frac{M-n+1}{N_{b} \beta\left\langle\alpha(p)\left|H_{1, b}\right| \alpha(p)\right\rangle} .
\end{aligned}
$$

(2) Directed loop update: We can construct the loop as follows. First, select randomly one of the vertex legs as an initial entrance leg. The exit vertex leg is chosen with the probability as Eq. (A17), and both the entrance and exit spins

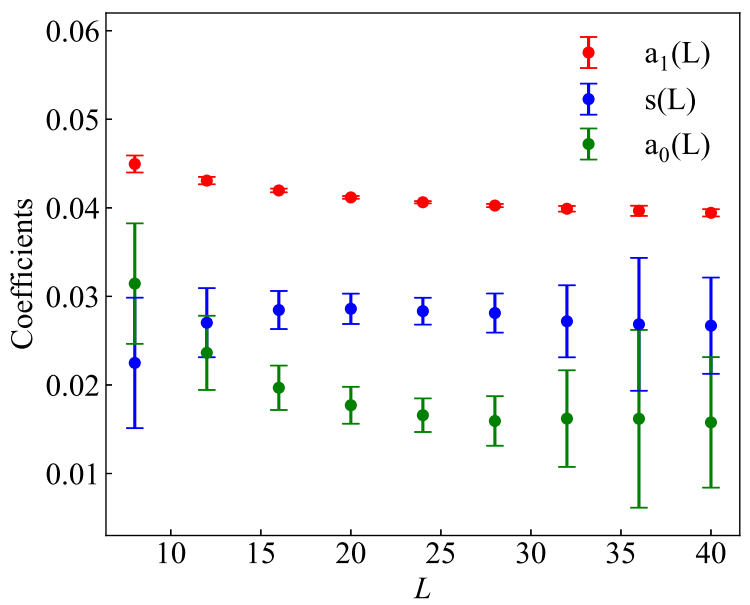

FIG. 10. Finite-size convergence of the coefficients of the disorder operator at the $(2+1) d$ Ising critical point, for the case of $M=R \times R$. 
are flipped. The probability of the exit leg is defined with matrix elements obtained by flipping spins in a vertex. The elements are defined as

$$
W\left(\begin{array}{l}
g_{3}, g_{4} \\
g_{1}, g_{2}
\end{array}\right)=\left\langle g_{3} S_{i}^{z}, g_{4} S_{j}^{z}\left|H_{b}\right| g_{1} S_{i}^{z}, g_{2} S_{j}^{z}\right\rangle,
$$

where $g_{i}=-1$ if the spin on leg $i$ is flipped and $g_{i}=+1$ if it is not flipped. For example, the probability of exiting at leg 3 if the entrance is at leg 1 is given by

$$
P_{3,1}=\frac{W\left(\left(_{-+}^{-+}\right)\right.}{W\left(\begin{array}{l}
++ \\
++
\end{array}\right)+W\left(\left(_{--}^{++}\right)+W\left(\left(_{-+}^{-+}\right)+W\left(\left(_{-+}^{+-}\right)\right.\right.\right.} .
$$

Then let it visit the next vertex. The loop goes on in this way one vertex by one until it closes. Also we use the Swendsen-Wang scheme to flip the clusters after all clusters are identified.

\section{Curve fitting}

Lastly, we show the details of fitting results of Figs. 6 and 7. We fit the disorder operator according to Eq. (21) and obtain the coefficients $a_{1}, s$, and $a_{0}$ for different system sizes. Figure 10 demonstrates the convergence of the fitting results as the system size increases.
[1] R. Moessner and S. L. Sondhi, Phys. Rev. B 63, 224401 (2001).

[2] S. V. Isakov and R. Moessner, Phys. Rev. B 68, 104409 (2003).

[3] T. Senthil, L. Balents, S. Sachdev, A. Vishwanath, and M. P. A. Fisher, Phys. Rev. B 70, 144407 (2004).

[4] Y.-Y. He, H.-Q. Wu, Y.-Z. You, C. Xu, Z. Y. Meng, and Z.-Y. Lu, Phys. Rev. B 93, 115150 (2016).

[5] N. Ma, Y.-Z. You, and Z. Y. Meng, Phys. Rev. Lett. 122, 175701 (2019).

[6] A. Altland and B. Simons, Condensed Matter Field Theory (Cambridge University Press, Cambridge, U.K., 2010).

[7] D. Poland, S. Rychkov, and A. Vichi, Rev. Mod. Phys. 91, 015002 (2019).

[8] X.-G. Wen, Science 363, eaal3099 (2019).

[9] E. Fradkin, Field Theories of Condensed Matter Physics (Cambridge University Press, Cambridge, U.K., 2013).

[10] K. Gregor, D. A. Huse, R. Moessner, and S. L. Sondhi, New J. Phys. 13, 025009 (2011).

[11] Z. Nussinov and G. Ortiz, Proc. Natl. Acad. Sci. USA 106, 16944 (2009).

[12] Z. Nussinov and G. Ortiz, Ann. Phys. 324, 977 (2009).

[13] D. Gaiotto, A. Kapustin, N. Seiberg, and B. Willett, J. High Energy Phys. 02 (2015) 172.

[14] W. Ji and X.-G. Wen, Phys. Rev. Res. 2, 033417 (2020).

[15] L. Kong, T. Lan, X.-G. Wen, Z.-H. Zhang, and H. Zheng, Phys. Rev. Res. 2, 043086 (2020).

[16] D. Gaiotto, A. Kapustin, Z. Komargodski, and N. Seiberg, J. High Energy Phys. 05 (2017) 091.

[17] F. Wegner, J. Math. Phys. 12, 2259 (1971).

[18] L. P. Kadanoff and H. Ceva, Phys. Rev. B 3, 3918 (1971).

[19] E. Fradkin, J. Stat. Phys. 167, 427 (2017).

[20] X.-C. Wu, W. Ji, and C. Xu, arXiv:2012.03976.

[21] Y.-C. Wang, M. Cheng, and Z. Y. Meng, arXiv:2101.10358.

[22] X.-C. Wu, C.-M. Jian, and C. Xu, arXiv:2101.10342.

[23] S. Gukov and E. Witten, arXiv:0804.1561.

[24] N. Ma, G.-Y. Sun, Y.-Z. You, C. Xu, A. Vishwanath, A. W. Sandvik, and Z. Y. Meng, Phys. Rev. B 98, 174421 (2018).

[25] X.-G. Wen, Phys. Rev. B 99, 205139 (2019).

[26] E. Lake, arXiv:1802.07747.

[27] D. M. Hofman and N. Iqbal, SciPost Phys. 6, 006 (2019).

[28] M. B. Hastings and X.-G. Wen, Phys. Rev. B 72, 045141 (2005).

[29] J. B. Kogut, Rev. Mod. Phys. 51, 659 (1979).
[30] M. Levin, Commun. Math. Phys. 378, 1081 (2020).

[31] W. Ji and X.-G. Wen, Phys. Rev. Res. 1, 033054 (2019).

[32] H. Casini and M. Huerta, Nucl. Phys. B 764, 183 (2007).

[33] H. Casini and M. Huerta, J. Phys. A: Math. Theor. 42, 504007 (2009).

[34] J. Dowker, arXiv:1509.00782.

[35] H. Elvang and M. Hadjiantonis, Phys. Lett. B 749, 383 (2015).

[36] J. Helmes, L. E. Hayward Sierens, A. Chandran, W. WitczakKrempa, and R. G. Melko, Phys. Rev. B 94, 125142 (2016).

[37] P. Bueno, H. Casini, and W. Witczak-Krempa, J. High Energy Phys. 08 (2019) 069.

[38] C. Berthiere, Phys. Rev. B 99, 165113 (2019).

[39] S. N. Solodukhin, Phys. Lett. B 665, 305 (2008).

[40] D. Fursaev, J. High Energy Phys. 05 (2012) 080.

[41] D. Gioev and I. Klich, Phys. Rev. Lett. 96, 100503 (2006).

[42] M. M. Wolf, Phys. Rev. Lett. 96, 010404 (2006).

[43] H.-H. Lai, K. Yang, and N. E. Bonesteel, Phys. Rev. Lett. 111, 210402 (2013).

[44] A. Paramekanti, L. Balents, and M. P. A. Fisher, Phys. Rev. B 66, 054526 (2002)

[45] T. Tay and O. I. Motrunich, Phys. Rev. Lett. 105, 187202 (2010).

[46] H. Casini, M. Huerta, and R. C. Myers, J. High Energy Phys. 05 (2011) 036.

[47] M. A. Metlitski, C. A. Fuertes, and S. Sachdev, Phys. Rev. B 80, 115122 (2009).

[48] H. W. J. Blöte and Y. Deng, Phys. Rev. E 66, 066110 (2002).

[49] Z. H. Liu, G. Pan, X. Y. Xu, K. Sun, and Z. Y. Meng, Proc. Natl. Acad. Sci. USA 116, 16760 (2019).

[50] A. W. Sandvik, Phys. Rev. E 68, 056701 (2003).

[51] O. F. Syljuåsen and A. W. Sandvik, Phys. Rev. E 66, 046701 (2002).

[52] O. F. Syljuåsen, Phys. Rev. E 67, 046701 (2003).

[53] Y. Da Liao, H. Li, Z. Yan, H.-T. Wei, W. Li, Y. Qi, and Z. Y. Meng, Phys. Rev. B 103, 104416 (2021).

[54] M. Billó, M. Caselle, D. Gaiotto, F. Gliozzi, M. Meineri, and R. Pellegrini, J. High Energy Phys. 07 (2013) 055.

[55] D. Gaiotto, D. Mazac, and M. F. Paulos, J. High Energy Phys. 03 (2014) 100.

[56] P. Bueno, R. C. Myers, and W. Witczak-Krempa, J. High Energy Phys. 09 (2015) 091. 